\title{
The Fidelity of $p 16$ Staining as a Surrogate Marker of Human Papillomavirus Status in Fine- Needle Aspirates and Core Biopsies of Neck Node Metastases: Implications for HPV Testing Protocols
}

\author{
Brittany J. Holmes ${ }^{\text {a }}$ Zahra Maleki $^{\text {a }}$ William H. Westra ${ }^{\mathrm{a}-\mathrm{c}}$ \\ Departments of a Pathology, ${ }^{\mathrm{b}}$ Oncology and ${ }^{\mathrm{C} O}$ Otolaryngology-Head and Neck Surgery, Johns Hopkins Medical \\ Institutions, Baltimore, Md., USA
}

\section{Key Words}

Aspiration biopsy - Fine-needle aspiration .

Human papillomavirus. Head and neck

\begin{abstract}
Objectives: The importance of detecting human papillomavirus (HPV) in head and neck squamous cell carcinoma (HN$\mathrm{SCC}$ ) has resulted in a growing expectation for HPV testing of clinical samples. Although testing protocols vary, most pertain to primary tumor biopsies/resections. Testing of fine-needle aspirates and core biopsies (FNACBs) is advantageous, but it is unclear whether technical and biological factors adversely affect the fidelity of HPV detection in these samples. Methods: Data was collected for 85 patients with regionally metastatic HNSCC that had undergone FNACB with HPV analysis as part of clinical care. HPV testing consisted of p16 immunostaining and HPV in situ hybridization (ISH). The FNACBs were compared with the subsequent biopsies/resections for HPV status. Results: p16 staining was present in 60 cases $(71 \%)$. p16 positivity was predictive of oropharyngeal origin $(p<0.001)$ and correlated with the presence of HPV by ISH ( $98 \%$ correlation). On comparison of the metastases and primary cancers, the HPV status was concordant in 58 of 59 cases (98\%). Conclusions: For patients
\end{abstract}

with metastatic HNSCC, p16 staining reliably reflects the HPV status of the primary tumor. p16 staining of FNACBs may obviate the need for more invasive sampling of the primary cancer solely for the purpose of HPV testing.

(c) 2015 S. Karger AG, Basel

\section{Introduction}

The human papillomavirus (HPV), particularly type 16 , has been established as an important cause of about $25 \%$ of head and neck squamous cell carcinomas (HNSCCs) overall and up to $80 \%$ of those HNSCCs arising in the oropharynx (OPSCCs) $[1,2]$. The identification of HPV in OPSCC identifies a distinct form of HNSCC that is highly responsive to various forms of therapy and is associated with a favorable clinical outcome [3-5]. Accordingly, there has been a move among cancer care organizations towards mandatory HPV testing for all patients with OPSCC. Based on a comprehensive meta-analysis of the medical literature, Cancer Care Ontario recently established guidelines for HPV testing that called for the following: (1) HPV testing of all OPSCCs, (2) HPV testing of metastatic squamous cell carcinomas to neck lymph nodes of patients with occult primary tumors and (3) ini-

\section{KARGER 125}

(c) 2015 S. Karger AG, Base

0001-5547/15/0591-0097\$39.50/0

E-Mail karger@karger.com

www.karger.com/acy
Correspondence to: Dr. William H. Westra

Johns Hopkins Hospital

Weinberg Building, Room 2242, 401 N. Broadway

Baltimore, MD 21231 (USA)

E-Mail wwestra@jhmi.edu 


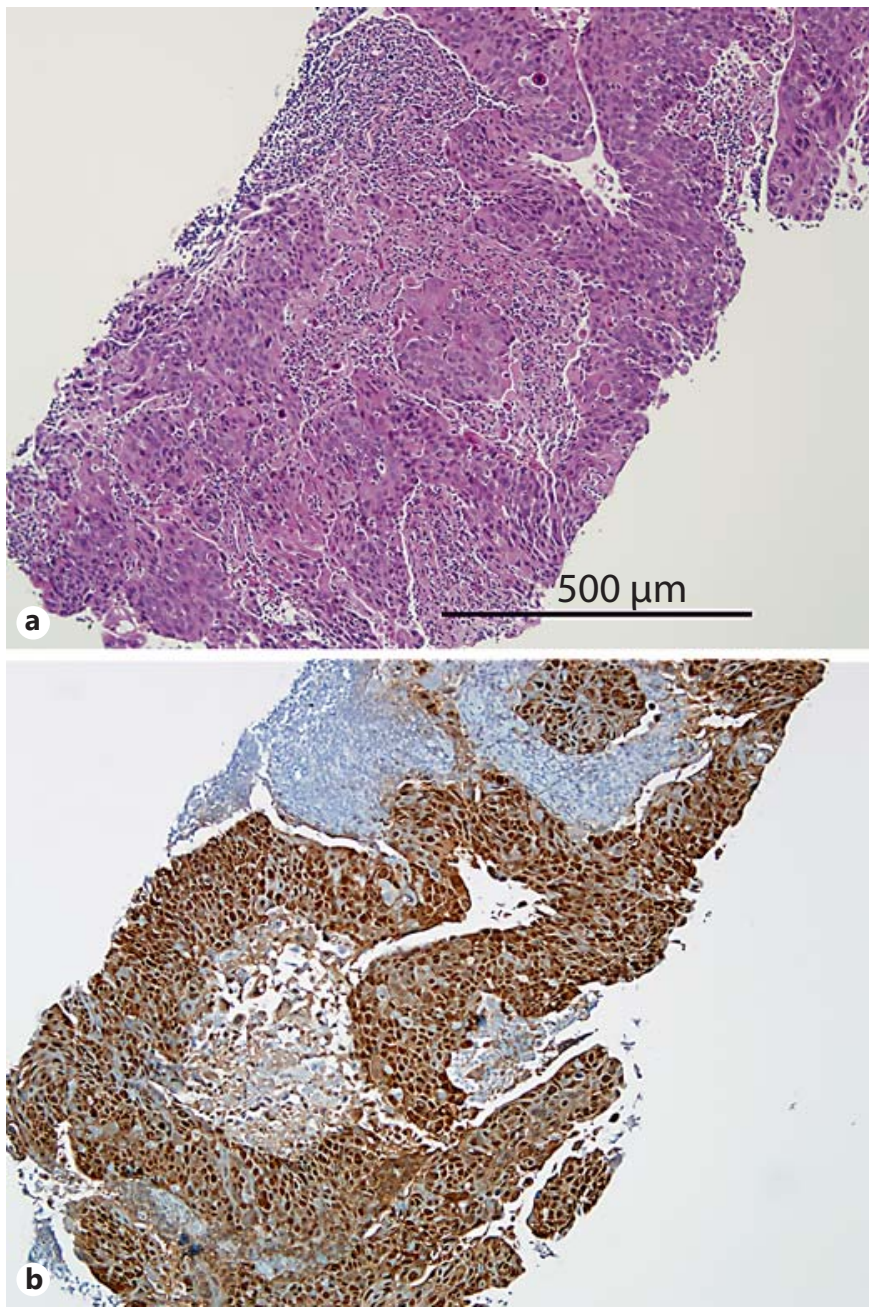

Fig. 1. Core needle biopsy of an oropharyngeal squamous cell carcinoma metastatic to a cervical lymph node. This type of sampling not only preserves the histological context (a; routine HE stain) but provides ample tissue for ancillary studies such as HPV testing (b; p16 immunohistochemical stain).

tial determination of HPV status in OPSCCs using p16 immunohistochemical staining (https:/www.cancercare. on.ca/common/pages/UserFile.aspx?fileId=279836). Although these recommendations are reasonable, they do not adequately address some of the more germane issues surrounding HPV testing in the clinical arena.

Patients commonly enter oncological care based on a neck mass diagnosed as metastatic HNSCC by cytopathological assessment. For these patients who have not yet had a tissue diagnosis of the carcinoma at its primary site, future guidelines must address practical concerns regarding the nature and sufficiency of HPV testing in fine-needle aspirates and core biopsies (FNACBs). Potentially, the use of these samples as a substrate for HPV assessment could clarify HPV tumor status and eliminate the requisite excisional tissue biopsy via a more aggressive surgical procedure. While the feasibility of p16 immunostaining in FNAs and biopsies of cervical lymph node metastases has been confirmed in a limited number of studies [6-8], p16 status of nodal metastases as a proxy for HPV status of the HNSCC at its primary site has not been confirmed in a comparative analysis. Toward this end, we analyzed our clinical experience of p16 immunostaining of FNACBs in patients presenting with nodal metastases in an effort to do the following: (1) confirm the specificity of p16 staining for oropharyngeal origin, (2) correlate p16 immunostaining with the actual presence of high-risk HPV as determined by HPV DNA and (3) establish the fidelity of p16 status across regional and primary sites.

\section{Patients and Methods}

\section{Cases}

Data were collected on all cases where, as part of clinical care at the Johns Hopkins Hospital, p16 staining was performed on FNAs of metastatic squamous cell carcinomas involving cervical lymph nodes. At our institution, it is now common practice to supplement these FNAs with core needle biopsies to enhance sample adequacy, and these core biopsies were also used for HPV analysis (fig. 1). The FNAs were ultrasound guided and performed using a 25 -gauge spinal needle, and the core biopsies were performed with a 20-gauge core biopsy device. Medical records, including the cytopathology and surgical pathology reports, were reviewed to document patient age and gender, tumor diagnosis, the primary site of tumor origin, and results of p16 staining and HPV DNA in situ hybridization (ISH). All pathology slides were reviewed, including the cytopathology slides and, when available, the histopathology slides taken of the subsequent tumor resections and biopsies. For those cases with incomplete data sets (e.g. p16 immunohistochemistry and/or HPV DNA ISH not originally performed on primary tumor resection), the blocks were retrieved to complete the HPV analysis.

\section{p16 Immunohistochemistry}

Tissue sections of $5 \mu \mathrm{m}$ were deparaffinized. Antigen retrieval was performed using heat-induced epitope retrieval with $10 \mathrm{mM}$ of citrate buffer. p16 expression was evaluated by the use of a mouse monoclonal antibody against p16 (MTM Laboratories, Heidelberg, Germany) visualized using the Ventana XT autostainer using the 1-view secondary detection kit (Ventana Medical Systems, Tucson, Ariz., USA). When dealing with p16 staining in tissue sections of HNSCC, the pattern of p16 expression is generally observed to be dichotomous. In other words, p16 staining is either absent (no staining) or present (moderate-to-strong diffuse nuclear and cytoplasmic staining). In equivocal cases, the presence of nuclear and cytoplasmic staining, even when faint, is strongly associated with the presence of HPV [9]. Accordingly, p16 expression was scored as positive if any cytoplasmic and nuclear staining 

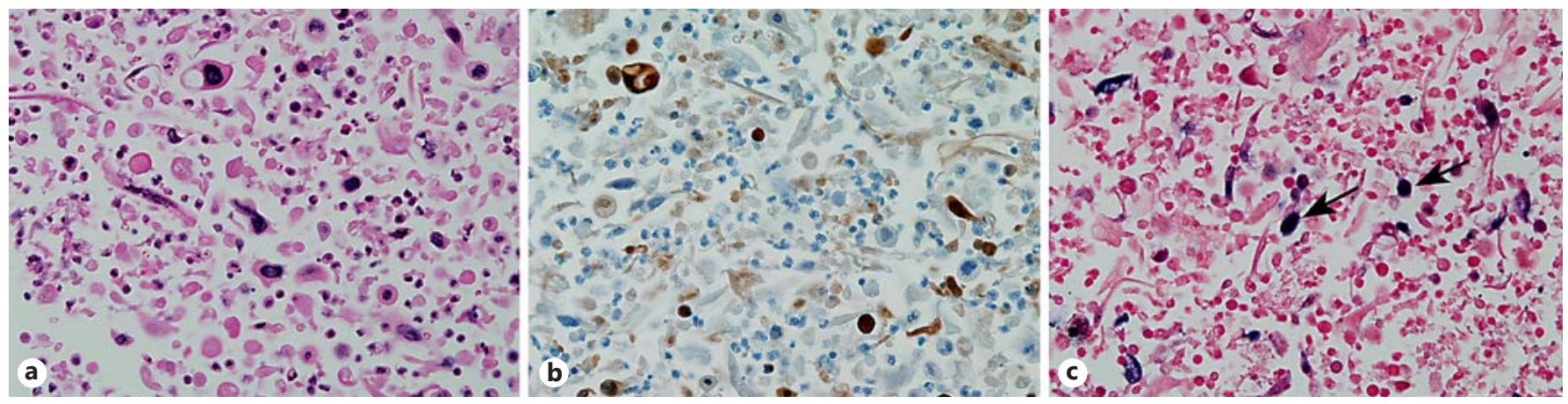

Fig. 2. FNA (cell block) of a metastatic OPSCC evaluated by routine HE staining (a), p16 immunostaining (b) and HPV ISH (c). Despite the presence of cell degradation, the tumor cells retain p16 immunoreactivity (b) and HPV 16 hybridization signals (c; arrows).

was observed in the FNACBs, even in the presence of cellular degradation where it was difficult to quantify the percentage of positive tumor cells (fig. 2). For the resected primary carcinomas, p16 expression was scored as positive if nuclear and cytoplasmic staining was present in $\geq 70 \%$ of the tumor specimen. An HPV 16-positive oropharyngeal cancer was used as a positive control.

\section{HPV DNA in situ Hybridization}

In this study, HPV DNA ISH rather than a PCR-based detection assay was used to confirm HPV status. ISH permits direct microscopic confirmation of the presence and distribution of HPV. In contrast, PCR-based assays are unable to determine whether viral DNA arises from the population of cancer cells or the surrounding nonneoplastic tissues, and they do not allow for the recognition of tumor-free samples and thus the identification of false-negative results.

Tissue sections $(5 \mu \mathrm{m})$ from the formalin-fixed paraffin-embedded tumor blocks were evaluated for the presence of HPV DNA by ISH. Two different detection methods were used. A type 16-specific assay was performed using the ISH-catalyzed signal amplification method for biotinylated probes (DAKO GenPoint, Carpinteria, Calif., USA). Briefly, the $5-\mu \mathrm{m}$ tissue sections underwent deparaffinization, heat-induced target retrieval in citrate buffer and digestion using Proteinase K (Roche Diagnostics, Indianapolis, Ind., USA). The slides were subsequently hybridized with a biotinylated HPV 16 type-specific probe (DAKO). Signal amplification was performed by the consecutive application of a streptavidin-HRP complex and AQ2 biotinyl tyramide. Visualization of hybridization signals was performed by incubation with the chromogenic substrate diaminobenzidine.

For broader high-risk HPV detection, we also used the Ventana Inform HPV III Family 16 probe (B) kit (Ventana Medical Systems). For this assay, slides were conditioned using Ventana cell conditioner 2 and ISH protease 3. Hybridization utilized the HPV III Family 16 probe set that captures high-risk HPV genotypes 16, $18,33,35,45,51,52,56$ and 66 . Signals were detected using the ISH iView Blue Plus Detection Kit, which is an indirect biotin-streptavidin system that detects fluorescein-labeled probes. The kit uses an alkaline phosphatase enzyme and NBT/BCIP substrate chromogen reaction that provides an intense blue, permanent color as well as a red counter stain. All reagents are provided prediluted and ready to use on BenchMark Series automated slide stainers (Ventana Medical Systems).

For both detection assays, punctate hybridization signals localized to the tumor cell nuclei defined an HPV-positive tumor (fig. 3). An HPV-16-positive oropharyngeal cancer and HPV16-positive $\mathrm{SiHa}$ and CaSki cell lines were used as positive controls.

\section{Results}

p16 staining was performed on lymph node aspirates and core biopsies from 85 patients with metastatic HNSCC; 71 patients were male (84\%). Patient ages ranged from 20 to 89 years (median 59 , mean 58 ). The primary sites of tumor origin were the oropharynx (tonsil, $n=31$; base of tongue, $\mathrm{n}=23$; soft palate, $\mathrm{n}=1)$, oral cavity $(\mathrm{n}=$ $7)$, larynx $(n=5)$, facial skin $(n=3)$, nasopharynx $(n=1)$ and lung $(n=1)$. In 13 cases $(15 \%)$ the primary site was unknown. Of these 85 specimens, 43 (51\%) were obtained by FNA (cell blocks, $n=42$; cytospin, $n=1$ ), and $42(49 \%)$ were obtained by core biopsy. In 5 cases, cellularity was inadequate for supplemental analysis by DNA ISH.

Positive p16 staining was present in 60 of the FNACBs (71\%). A summary of p16 staining as a function of tumor site and HPV status is provided in figure 4 . By the site of the primary tumor origin, p16 staining was noted in 51 of the 55 lymph node metastases from the oropharynx (93\%), 7 of 13 metastases from unknown primary sites (54\%) and 2 of 17 metastases from nonoropharyngeal sites $(13 \%)$. For those patients with metastatic squamous cell carcinomas of known primary site, p16 positivity was strongly predictive of oropharyngeal origin (93 vs. 13\%, $\mathrm{p}<0.001$, Fisher's exact test). 
Fig. 3. HPV status is consistently maintained across stages of tumor progression. The FNA is shown on the left $(\mathbf{a}-\mathbf{c})$, and the resection of the primary oropharyngeal carcinoma is shown on the right $(\mathbf{d}-\mathbf{f})$. Both samples show p16 staining (b, e) and the presence of DNA ISH signals (c, $\mathbf{f}$; Ventana Inform HPV III Family 16 Probe and DAKO HPV 16 type-specific probe, respectively).

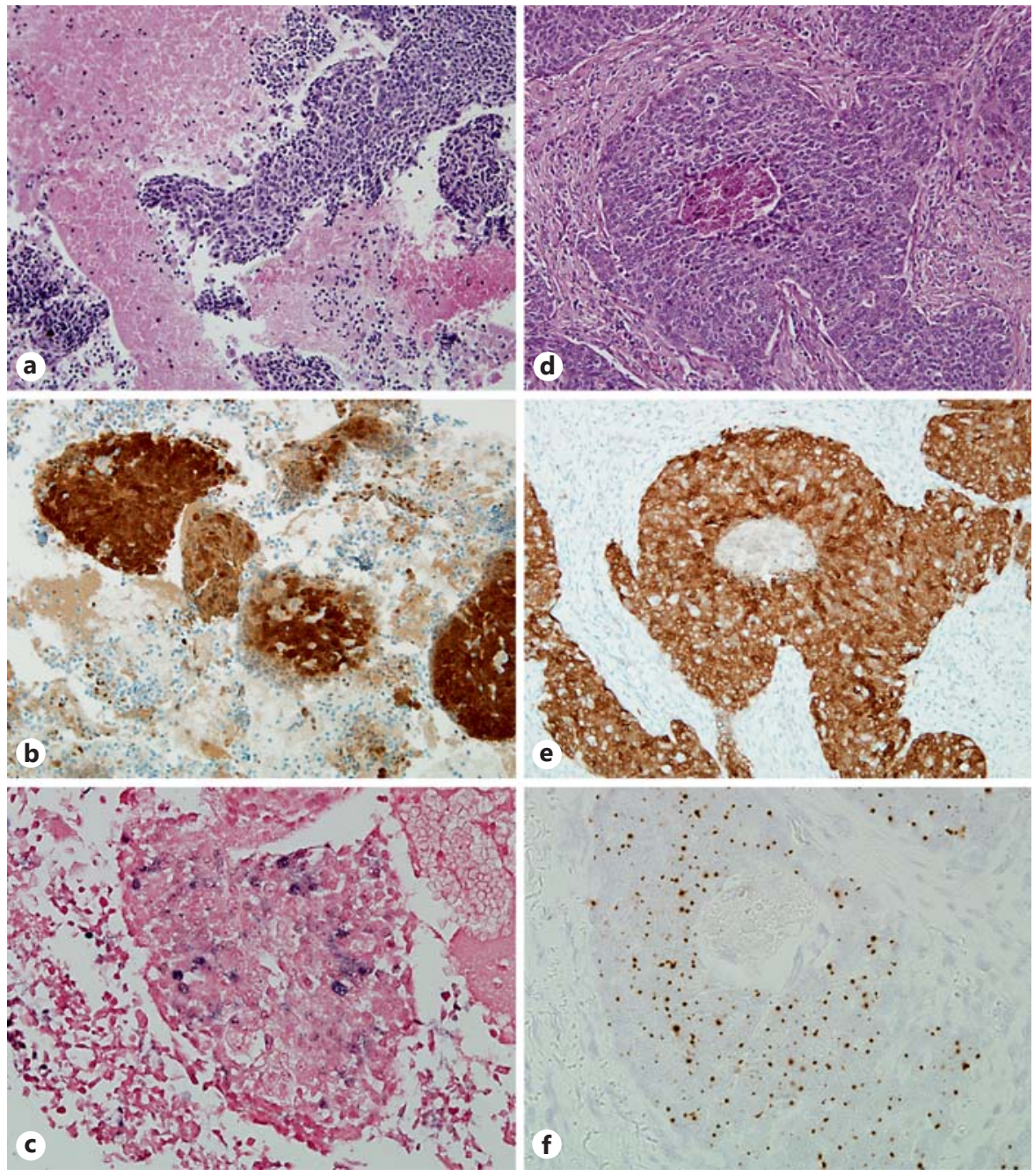

When dealing with FNACBs of metastatic HNSCCs, p16 staining helps identify not only the site of tumor origin but also the presence of HPV [10]. There was a 98\% correlation between HPV ISH and p16 staining, confirming p16 staining as a reliable surrogate marker for the presence of HPV (fig. 4). HPV DNA ISH was not performed in 5 cases (4 from oropharynx, 1 from unknown primary) due to insufficient cellularity or cellular degradation. In the FNACBs of sufficient cellularity and cellular integrity, HPV ISH signals were detected in 55 samples $(69 \%)$ and not detected in 25 samples (31\%). There was $98 \%$ correlation between HPV ISH and p16 staining. All 55 HPV ISH-positive cases were p16 positive, and 23 HPV ISH-negative cases demonstrated the absence of p16 staining. The 2 discordant cases ( 16 positive/HPV ISH negative) were from OPSCCs that were $\mathrm{p} 16$ positive/ HPV ISH negative $(n=1)$ and $\mathrm{p} 16$ positive/HPV ISH pos- itive $(\mathrm{n}=1)$ based on HPV analysis of the resection specimens.

To establish the fidelity of p16 status across primary and metastatic sites, we also evaluated the HPV status of the primary tumor resections and biopsies. A comparison of HPV status is summarized in table 1. A surgical pathology specimen (excisional biopsy or resection) was available for 59 of the paired primary HNSCCs (69\%). There was $98 \%$ concordance. The single discrepant case was an HPV-positive/p16-positive tonsillar carcinoma where the FNACB was p16 negative. On retrospective review of this discordant case, the material in the FNA cell block was suboptimal due to scant cellularity. For HPV-positive carcinomas, the HPV status was consistently retained through progression to regional nodal spread, and this retention was reliably confirmed in p16 assessment of the FNACBs (fig. 3; table 1). 


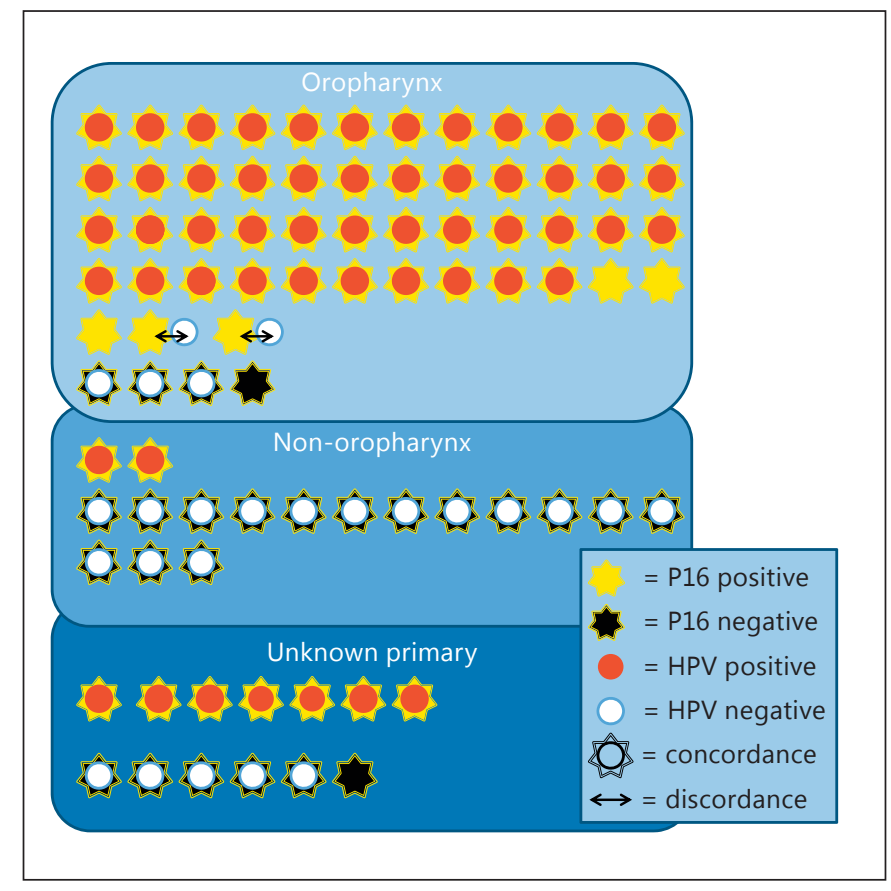

Fig. 4. p16 immunostaining of metastatic HNSCCs showing the distribution of staining by tumor origin and as a function of HPV ISH. Each star (p16 staining) and corresponding circle (ISH) represent results from individual FNA/biopsy samples. Superimposed symbols reflect concordant results, whereas nonoverlapping symbols (double-sided arrows) reflect discordant results. In 5 cases, p16 staining was performed alone without corresponding ISH analysis. p16-positive metastases tended to be from the oropharynx and tended to be HPV positive by ISH.

\section{Discussion}

Current guidelines have called for routine HPV assessment for OPSCCs. Nodal metastases have also been identified as an appropriate substrate for HPV analysis but only for those patients with clinically occult primary tumors. For patients with nodal metastasis from a known oropharyngeal mass, it is not known whether nodal metastases can reliably substitute for the primary tumor when establishing HPV status. Potentially, a number of biological and technical factors could drive HPV discordance at these two sites, including the following: (1) the timing (early vs. late) and necessity (transient vs. obligatory) of HPV infection during clinical tumor progression, (2) tumor degradation in lymph node metastases and (3) insufficient tumor sampling afforded by FNA biopsy.

Based on clinical HPV testing of tissue samples, over $80 \%$ of OPSCCs are HPV positive [2]. We were able to detect p16 staining in the vast majority (93\%) of meta-
Table 1. Comparison of HPV status for primary HNSCCs and corresponding lymph node metastases using p16 immunohistochemistry and HPV ISH

\begin{tabular}{|c|c|c|c|c|c|}
\hline \multirow{2}{*}{$\begin{array}{l}\text { Metastatic } \\
\text { FNACBs }\end{array}$} & \multicolumn{5}{|c|}{ Primary tumor resections } \\
\hline & $\begin{array}{l}\text { p16+l } \\
\text { HPV+ }\end{array}$ & $\begin{array}{l}\text { p16-I } \\
\text { HPV- }\end{array}$ & $\begin{array}{l}\text { p16+l } \\
\text { HPV- }\end{array}$ & $\begin{array}{l}\text { p16+l } \\
\text { HPV ND }\end{array}$ & $\begin{array}{l}\text { p16-/ } \\
\text { HPV ND }\end{array}$ \\
\hline $\mathrm{p} 16+/ \mathrm{HPV}+$ & 38 & 0 & 0 & 0 & 0 \\
\hline p16-/HPV- & 0 & 17 & 0 & 0 & 1 \\
\hline p16+/HPV ND & 2 & 0 & 0 & 0 & 0 \\
\hline p16-/HPV ND & 1 & 0 & 0 & 0 & 0 \\
\hline
\end{tabular}

Concordance rate $=98 \% . \mathrm{ND}=$ Not done.

static oropharyngeal squamous cell carcinomas in patients who had undergone FNACBs of involved regional lymph nodes. In effect, technical factors that could potentially influence p16 staining in FNACBs such as cystic degeneration and limited sampling did not reduce the expected high rate of p16 positivity. Conversely, the high rate of p16 staining did not reflect nonspecific staining or an inappropriately low threshold for p16 detection. In contrast to metastases from the oropharynx, p16 staining was not as commonly encountered in metastases from nonoropharyngeal sites ( 93 vs. $13 \%, \mathrm{p}<0.001$ ). This preferential distribution of p16 staining for carcinomas of the oropharynx supports the use of p16 staining as a staging tool in discerning the site of tumor origin for those patients presenting with cervical lymph node metastases $[7$, $8,11]$. Immunostaining for p16 protein has recently been regarded as a practical alternative or complementary procedure for HPV testing of oropharyngeal carcinomas based on a high correlation between HPV detection and p16 overexpression in tissue-based studies [10, 12-14]. We found that the use of p16 staining as a surrogate marker of HPV status is also reliable and valid for FNACBs, even in specimens with marked cellular degeneration. In the 80 cases where the FNACBs were evaluated using both methods, all but 2 cases were concordant for an overall concordance rate of $98 \%$. This strong correlation, together with the simplicity, low cost and high sensitivity of p16 immunocytochemistry suggests that p16 staining could function as a stand-alone test in the evaluation of FNACBs, at least in its role as a prognostic indicator.

HPV has been detected in dysplasia of the tonsillar crypts, and its presence in these early lesions has been taken to support its biological role as a relevant initiator of tumorigenesis [15]. We found that the HPV status of 
primary HNSCCs is consistently retained in regional lymph node metastases, supporting the critical role of HPV in the maintenance of the malignant phenotype. Indeed, the presence of transcriptionally active HPV across all stages of clinical progression, including distant metastases long after treatment of the primary tumor, dispels any notion of a 'hit-and-run' mechanism where the presence of HPV becomes superfluous over time [16-18]. The fidelity of HPV status across temporally and spatially separated lesions is of considerable practical significance. For the squamous cell carcinoma in the lung of a patient with a prior HPV-related OPSCC, HPV detection provides a direct link between the two tumors and provides a useful tool for discriminating between lung metastases and new lung primaries $[17,19]$. Most patients with HNSCC already have metastatic spread to regional lymph nodes at the time of presentation, about $15 \%$ of patients present with a neck mass as the first and only clinical manifestation and 3-9\% of the primary tumors elude detection despite a thorough clinical, radiographic, endoscopic, and histopathological evaluation [20]. FNACB represents an initial step in the evaluation of these patients. In light of the high fidelity of HPV status across stages of clinical progression, the exploitation of these FNACB samples as a substrate for HPV assessment could facilitate the diagnosis of a HPV-related HNSCC, direct the search for site of origin, predict clinical outcome, and guide therapy, all while abrogating the need for tissue acquisition of the primary tumor via a surgical procedure.

The few studies that have addressed HPV testing of cytological samples have primarily tried to adapt tissuetargeted approaches (e.g. p16 immunohistochemistry and HPV ISH) to archived cytological specimens [6-8]. In most instances, HPV testing of cytological specimens is restricted to a small subset of cases in which ample cel- lular material is available for the construction of cell blocks. In a prior analysis of FNA specimens, only $20 \%$ of the clinical samples were sufficiently cellular for the construction of cell blocks for HPV testing [7]. At the Johns Hopkins Hospital, FNA is now routinely augmented by the supplemental core needle biopsy in an effort to enhance sample adequacy for HPV testing of metastatic HNSCCs. The core needle biopsy has been advocated as a complementary method for sampling head and neck lesions in patients undergoing FNAs [21, 22]. Like the FNA, it is minimally invasive, cost-effective and safe. Specifically, the risk of major bleeding and tumor cell displacement ('seeding') is very low [22]. Although the core biopsy may not significantly improve the overall sensitivity of diagnosing squamous cell carcinoma in lymph node metastasis, it has an immense impact on sample adequacy. In a meta-analysis of core needle biopsies used in the evaluation of head and neck lesions, adequate material for histological diagnosis and ancillary studies was obtained in $95 \%$ of cases [22]. The development of liquid phase assays (e.g. Hybrid Capture $2[23,24]$ and Cervista ${ }^{\circledR}[25$, 26]) has recently been impelled by a need to circumvent the limiting prerequisite for high cellularity, but until these assays are validated in larger studies the FNA supplemented by the core needle biopsy provides a practical approach for determining the HPV status of metastatic HNSCCs. Indeed, the high fidelity of this approach would seem to render the need for additional tissue sampling of the primary tumor superfluous.

\section{Acknowledgment}

This work has been funded by the National Institute of Dental and Craniofacial Research (P50 DE019032).

\section{References}

1 Gillison ML, Koch WM, Capone RB, Spafford M, Westra WH, Wu L, Zahurak ML, Daniel RW, Viglione M, Symer DE, Shah KV, Sidransky D: Evidence for a causal association between human papillomavirus and a subset of head and neck cancers. J Natl Cancer Inst 2000;92:709-720.

$\checkmark 2$ Singhi AD, Westra WH: Comparison of human papillomavirus in situ hybridization and p16 immunohistochemistry in the detection of human papillomavirus-associated head and neck cancer based on a prospective clinical experience. Cancer 2010;116:2166-2173.
3 D’Souza G, Kreimer AR, Viscidi R, Pawlita M, Fakhry C, Koch WM, Westra WH, Gillison ML: Case-control study of human papillomavirus and oropharyngeal cancer. N Engl J Med 2007;356:1944-1956.

- 4 Ang KK, Harris J, Wheeler R, Weber R, Rosenthal DI, Nguyen-Tan PF, Westra WH, Chung CH, Jordan RC, Lu C, Kim H, Axelrod R, Silverman CC, Redmond KP, Gillison ML: Human papillomavirus and survival of patients with oropharyngeal cancer. N Engl J Med 2010;363:24-35.

\footnotetext{
5 Fakhry C, Westra WH, Li S, Cmelak A Ridge JA, Pinto H, Forastiere A, Gillison ML: Improved survival of patients with human papillomavirus-positive head and neck squamous cell carcinoma in a prospective clinical trial. J Natl Cancer Inst 2008;100: 261-269.

6 Umudum H, Rezanko T, Dag F, Dogruluk T: Human papillomavirus genome detection by in situ hybridization in fine-needle aspirates of metastatic lesions from head and neck squamous cell carcinomas. Cancer 2005; 105 : 171-177.
} 
7 Begum S, Gillison ML, Nicol TL, Westra WH: Detection of human papillomavirus-16 in fine-needle aspirates to determine tumor origin in patients with metastatic squamous cell carcinoma of the head and neck. Clin Cancer Res 2007;13:1186-1191.

8 Zhang MQ, El-Mofty SK, Davila RM: Detection of human papillomavirus-related squamous cell carcinoma cytologically and by in situ hybridization in fine-needle aspiration biopsies of cervical metastasis: a tool for identifying the site of an occult head and neck primary. Cancer 2008;114:118-123.

-9 Chen ZW, Weinreb I, Kamel-Reid S, PerezOrdonez B: Equivocal p16 immunostaining in squamous cell carcinoma of the head and neck: staining patterns are suggestive of HPV status. Head Neck Pathol 2012;6:422-429.

10 Lewis JS Jr: P16 immunohistochemistry as a standalone test for risk stratification in oropharyngeal squamous cell carcinoma. Head Neck Pathol 2012;6(suppl 1):S75-82.

-11 Begum S, Gillison ML, Ansari-Lari MA, Shah K, Westra WH: Detection of human papillomavirus in cervical lymph nodes: a highly effective strategy for localizing site of tumor origin. Clin Cancer Res 2003;9:6469-6475.

12 Hoffmann M, Ihloff AS, Gorogh T, Weise JB, Fazel A, Krams M, Rittgen W, Schwarz E, Kahn T: P16(INK4a) overexpression predicts translational active human papillomavirus infection in tonsillar cancer. Int J Cancer 2010; 127:1595-1602.

13 Jordan RC, Lingen MW, Perez-Ordonez B, He X, Pickard R, Koluder M, Jiang B, Wakely P, Xiao W, Gillison ML: Validation of methods for oropharyngeal cancer HPV status determination in US cooperative group trials. Am J Surg Pathol 2012;36:945-954.
14 El-Naggar AK, Westra WH: P16 expression as a surrogate marker for HPV-related oropharyngeal carcinoma: a guide for interpretative relevance and consistency. Head Neck 2012; 34:459-461.

15 Begum S, Cao D, Gillison M, Zahurak M, Westra WH: Tissue distribution of human papillomavirus 16 DNA integration in patients with tonsillar carcinoma. Clin Cancer Res 2005;11:5694-5699.

16 Ruzevick J, Olivi A, Westra WH: Metastatic squamous cell carcinoma to the brain: an unrecognized pattern of distant spread in patients with HPV-related head and neck cancer. J Neurooncol 2013;112:449-454.

17 Bishop JA, Ogawa T, Chang X, Illei PB, Gabrielson E, Pai SI, Westra WH: HPV analysis in distinguishing second primary tumors from lung metastases in patients with head and neck squamous cell carcinoma. Am J Surg Pathol 2012;36:142-148.

18 Mehrad M, Zhao H, Gao G, Wang X, Lewis JS Jr: Transcriptionally active human papillomavirus is consistently retained in the distant metastases of primary oropharyngeal carcinomas. Head Neck Pathol 2014;8:157-163.

19 Weichert W, Schewe C, Denkert C, Morawietz L, Dietel M, Petersen I: Molecular HPV typing as a diagnostic tool to discriminate primary from metastatic squamous cell carcinoma of the lung. Am J Surg Pathol 2009;33: 513-520.

20 de Braud F, al-Sarraf M: Diagnosis and management of squamous cell carcinoma of unknown primary tumor site of the neck. Semin Oncol 1993;20:273-278.
21 Witt BL, Schmidt RL: Ultrasound-guided core needle biopsy of salivary gland lesions: a systematic review and meta-analysis. Laryngoscope 2014;124:695-700.

22 Novoa E, Gurtler N, Arnoux A, Kraft M: Role of ultrasound-guided core-needle biopsy in the assessment of head and neck lesions: a meta-analysis and systematic review of the literature. Head Neck 2012;34:1497-1503.

23 Smith DF, Maleki Z, Coughlan D, Gooi Z, Akpeng B, Ogawa T, Bishop JA, Frick KD, Agrawal N, Gourin CG, Ha PK, Koch WM, Richmon JD, Westra WH, Pai SI: Human papillomavirus status of head and neck cancer as determined in cytologic specimens using the hybrid-capture 2 assay. Oral Oncol 2014; 50:600-604.

24 Bishop JA, Maleki Z, Valsamakis A, Ogawa T, Chang X, Pai SI, Westra WH: Application of the hybrid capture 2 assay to squamous cell carcinomas of the head and neck: a convenient liquid-phase approach for the reliable determination of human papillomavirus status. Cancer Cytopathol 2012;120:18-25.

25 Solomides CC, Bibbo M, Wang ZX: Assessment of fine-needle aspiration specimen adequacy for high-risk HPV detection and genotyping in oropharyngeal squamous cell carcinoma. Acta Cytol 2012;56:196-198.

26 Guo M, Khanna A, Dhillon J, Patel SJ, Feng J, Williams MD, Bell DM, Gong Y, Katz RL, Sturgis EM, Staerkel GA: Cervista HPV assays for fine-needle aspiration specimens are a valid option for human papillomavirus testing in patients with oropharyngeal carcinoma. Cancer Cytopathol 2014;122:96-103. 